\title{
A Zen Garden Interface for the Interactive Control of Sonic Ambiences in Smart Environment
}

\author{
Jiajun Yang, Thomas Hermann \\ Ambient Intelligence Group, CITEC, Bielefeld University \\ Inspiration 1, 33619 Bielefeld, Germany \\ E-mail: \{jyang, thermann\}@techfak.uni-bielefeld.de
}

Abstract

\begin{abstract}
In this paper we introduce SoZen, a novel interface for parameterizing aspects of ambient soundscapes including ambient music and peripheral sonifications using a Zen Garden. The SoZen system is both a stylish and aesthetic artifact in daily living environments and a computer-vision-based tangible and malleable representation for sound features. Due to the immediate correspondence between the arrangement of stones and the shape of sand with sonic features it also becomes a persistent visualization of the current sonic ambience. Thus it offers a conceptually different and 'calm' way of reviewing and specifying sounds. The paper focuses on the conceptual ideas and showcases our current system. We demonstrate some initial ambiences and conclude with current ideas on how to embed and evaluate SoZen in the context of a smart apartment environment.
\end{abstract}

Keywords-Cross-modal interaction, ambient soundscape, image feature extraction.

\section{INTRODUCTION}

Sound is a powerful carrier of contextual information and as subconsciously processed soundscape - also affects the listeners mood and mental state [1]. However, we rarely attend to or design our soundscapes, and mainly focus interior design on visual artifacts. Sound is often merely considered as selecting music, and the interfaces for that are often explicit, cognitive, attention-absorbing and rigid. This gives rise to the search for alternative means to give inhabitants ways to shape or refine the room's soundscape.

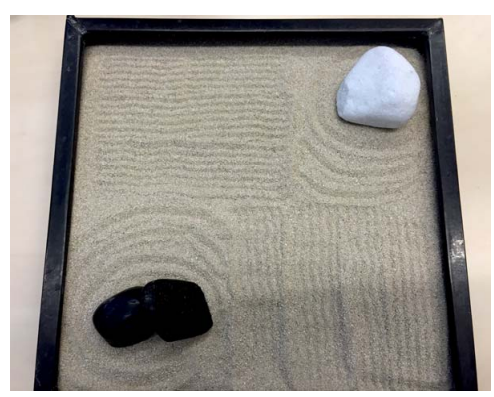

Fig. 1. Miniature Zen Garden used for our SoZen system.

SoZen is a multimodal installation that aims at producing non-intrusive ambient soundscapes in daily living environment. Users can initiate the soundscape by creating visual patterns on a miniature Zen Garden (or Japanese rock garden) (Fig. 1). The miniature Zen Garden is a small 'sand plate' to imitate the famous Japanese Buddhism gardens that use sands and stones in different patterns to represent various meaning of elements and metaphors [2]. The generated soundscape is a result of the cross-modal binding [3] based on the visual structure.

Using image to generate soundscape is not only a novel ways of cross-modal interaction, but also provides interesting interpretation between modals. Similar projects can usually be found in an exhibition environment (with literature examples). Visual feature analysis and sonification are explored in [4], in which fingerprint features were extracted to sonic parameters as a sonification approach. [5] presented an interactive music composition platform via paper drawing.

SoZen provides a novel canvas for users to create expressive patterns, which lead to corresponding soundscapes.

\section{PROJECT DESCRIPTION}

\section{A. Installation Overview}

The miniature Zen Garden is a representation of "mountainriver" (山水) in oriental culture, where the mountain is mimicked by stone and the river is represented by the shape of the sand. Therefore, a good first approach is to generate the soundscape according to the shapes of these two elements.

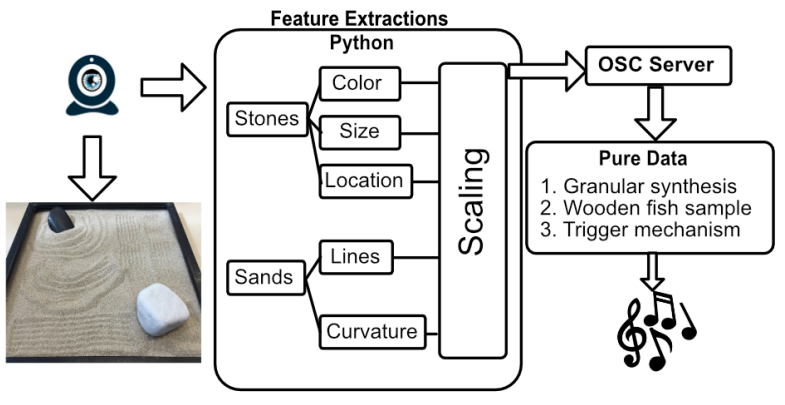

Fig. 2. System architecture

The system structure is presented in Figure 2: a webcam is used to capture the image of the sand plate. The feature analysis is executed in Python using OpenCV. The image features are then sent to Pure Data (PD) [6] via Open Sound 
Control (OSC) to control the audio parameters. A user only needs to 'decorate' the Zen Garden and the soundscape will automatically start to play back after the user finishes the visual composition.

\section{B. Image Feature Detection}

The stones (mountains) and sands (rivers) are the key elements of a Zen Garden, thus the feature detection focuses on the shapes of these two elements.

Regarding the stones, properties such as size, color and location are detected through blob detection methods. The analysis of the sand patterns is based on two aspects: lines and curvatures. The program detects the amount of lines and curvatures using the Hough Transform (see Fig. 3b for lines), which represents the hardness and softness of the shapes.

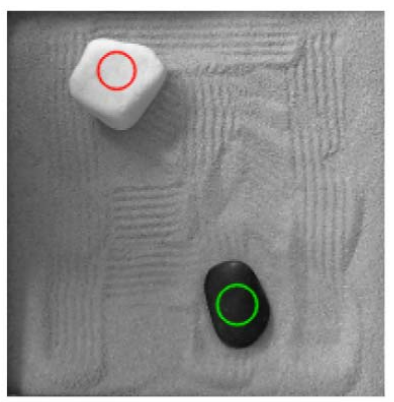

(a) Stone Detection

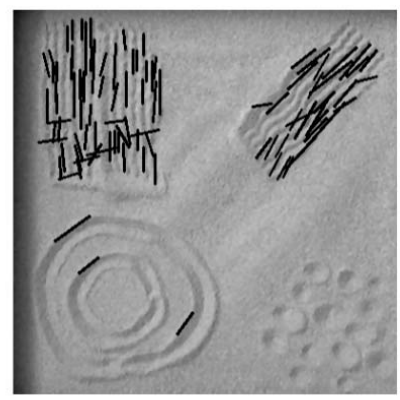

(b) Line Detection

Fig. 3. Examples of the current feature extractions

\section{Living Room Soundscape}

Soundscapes have the ability to support calmness, relaxation, also emotional state [7]. The soundscape created in SoZen ought to be sparse and non-intrusive, yet capable of interpreting the emotion from the shape of the garden.

The soundscape design is developed on Pure Data. We used a granular synthesizer to generate a pad-based ${ }^{1}$ soundscape based on a selection of samples, which include object and environmental sounds. Figure 4 depicts an example of six different samples being fed to the granular synthesis together. The spectral content becomes more complex from left to right. Hence, the granular pad will also sound more intense from left to right. The sand feature will then be used to decide the starting position of each grain in this sample space.

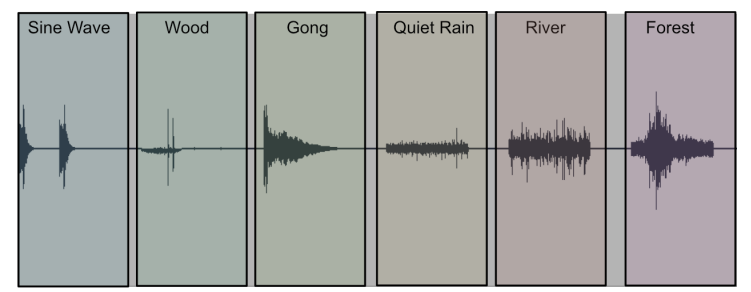

Fig. 4. Example of a soundscape region

1 A synthesis sound commonly used as a background element in music.
In addition to the granular synthesis, there is a sample of hitting a wooden fish ${ }^{2}$. The interval each trigger depends the amount of visual contents on the sand plate, where the more content will lead to more frequent occurrences.

A few audio examples can be downloaded from the link below:

https://www.dropbox.com/sh/iaz5sjgmnvay5rf/AAAkjSSOj I6e9hS7 -BrI-YZa?dl=0

\section{SUMMARY}

At the current stage, the sound design is nearly completed. The initial feature detections are presented in this paper. However, this abstract does not present a concrete interaction due to its early stage. For the next stage of the project, we aim at adopting more prominent feature extraction methods, possibly machine learning methods as the sand patterns are hard to be detected due to their subtleness. We also plan to continue the development of the mapping and sound design.

For evaluation, the installation will be placed in several offices in the department to accompany people's daily work. We plan to carry out a survey to study the experience of the soundscapes, especially whether the soundscape and visual patterns are matched cohesively, and whether the soundscape positively affect people's calmness and emotional state.

In summary, this abstract provides an overview of SoZen, the image data-driven soundscape interface via Zen Garden. The project is designed to be used in a living room or workspace environment to provide aesthetically pleasant ambient sounds that are consistent with the Zen Garden's state.

\section{ACKNOWLEDGMENT}

We wish to thank the German Research Foundation (DFG) for funding this project.

\section{REFERENCES}

[1] M. M. Bradley and P. J. Lang, "Affective reactions to acoustic stimuli," Psychophysiology, vol. 37, pp. 204-215, 2000.

[2] S. McGovern, "The Ryoan-ji Zen garden: textual meanings in topographical form," Visual Communication, vol. 3, no. 3, pp. 344-359, 2004.

[3] A. Tsiros, "Evaluating the perceived similarity between audio-visual features using corpus-based concatenative synthesis," Proceedings of the International Conference on New Interfaces for Musical Expression, 2014.

[4] Y. C. Han, B. Han and M. Wright, "Digiti Sonus: advanced interactive fingerprint sonification using visual feature analysis," Proceedings of the International Conference on New Interfaces for Musical Expression, 2013.

[5] T. Tsandilas, C. Letondal and W. Mackay, "Musink: composing music through augmented drawing," Proceedings of the SIGCHI Conference on Human Factors in Computing Systems, ACM, 2009.

[6] Pure Data, https://puredata.info/

[7] B. Mauney and B. Walker, "Creating functional and livable soundscape for peripheral monitoring of dynamic data," Proceedings of International Conference on Auditory Display, 2004.

\footnotetext{
2 A percussive instrument used for keeping the rhythm during sutra chanting.
} 